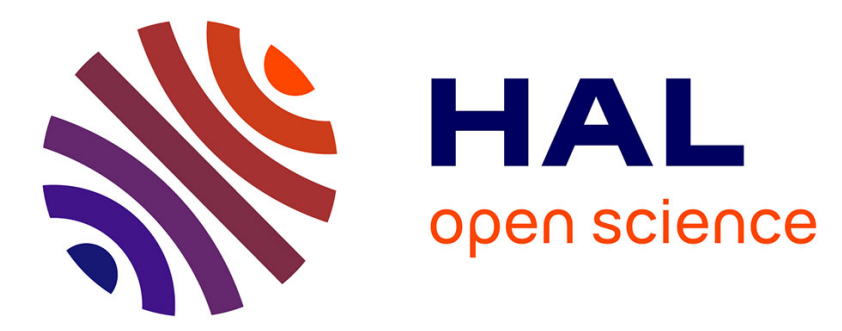

\title{
Self-Synchronization and Self-Stabilization of Walking Gaits Modeled by the Three-Dimensional LIP Model
}

Qiuyue Luo, Anne Kalouguine, Christine Chevallereau, Yannick Aoustin, Victor de Leon Gomez

\section{- To cite this version:}

Qiuyue Luo, Anne Kalouguine, Christine Chevallereau, Yannick Aoustin, Victor de Leon Gomez. Self-Synchronization and Self-Stabilization of Walking Gaits Modeled by the ThreeDimensional LIP Model. IEEE Robotics and Automation Letters, 2018, 3 (4), pp.3332 - 3339. 10.1109/lra.2018.2852767 . hal-01925444

\section{HAL Id: hal-01925444 \\ https://hal.science/hal-01925444}

Submitted on 29 Nov 2018

HAL is a multi-disciplinary open access archive for the deposit and dissemination of scientific research documents, whether they are published or not. The documents may come from teaching and research institutions in France or abroad, or from public or private research centers.
L'archive ouverte pluridisciplinaire HAL, est destinée au dépôt et à la diffusion de documents scientifiques de niveau recherche, publiés ou non, émanant des établissements d'enseignement et de recherche français ou étrangers, des laboratoires publics ou privés. 


\title{
Self-synchronization and Self-stabilization of Walking Gaits Modeled by the 3D LIP Model
}

\author{
Qiuyue Luo ${ }^{1}$, Victor De-León-Gómez ${ }^{1}$, Anne Kalouguine ${ }^{1,2}$, Christine Chevallereau $^{1}$, and Yannick Aoustin ${ }^{1}$
}

\begin{abstract}
The linear inverted pendulum (LIP) model is often used to study walking gaits, but the transition from one step to the following step is often neglected, while it is really important for the walking stability. This paper studies different landing positions of the swing foot, and different conditions to switch the stance leg, based on time or on the configuration of the robot. It is shown that self-synchronization of the motion in sagittal and frontal planes is dependent on different switching conditions. Neither self-synchronization nor self-stabilization is observed when the stance leg switching is based on time or when both the step length and width are fixed. On the other hand, selfsynchronization can be obtained when the switching condition of the stance leg is based on a linear combination of the positions of the center of mass (CoM) along the sagittal and frontal axes. Moreover, self-stabilization can be obtained when the velocity of the CoM in the sagittal plane is taken into account.
\end{abstract}

Index Terms-Humanoid and Bipedal Locomotion, Passive Walking, Dynamic stability

\section{INTRODUCTION}

A S humanoid robots are very complex 3D systems, many simplified models have been proposed for better understanding of their dynamic behaviors. The linear inverted pendulum (LIP) model proposed by S. Kajita [1] is often used to study walking gaits. This model assumes that the vertical acceleration of the CoM is zero, consequently an analytical expression to define the CoM evolution exists and equations in sagittal and frontal planes are decoupled.

In order to generate a periodic walking gait, the condition of transition from one step to the following step has to be defined. A large number of papers assume a constant period [2], [3], in which a preview over several steps is considered for gait planning. Englsberger et al. [4] proposed a gait pattern generator based on capture point (CP) [5] or divergent component of motion (DCM) [6], and this result was extended to 3D in [7]. In [8], an approach combining step location and timing adjustment was proposed for generating robust gaits. In these works, stable walking is based on the prediction of future states of the CoM and foot locations by evaluating the analytical dynamic model along the step. Our

Manuscript received: February, 21, 2018; Revised May, 27, 2018; Accepted June, 17, 2018.

This paper was recommended for publication by Editor Nikos Tsagarakis upon evaluation of the Associate Editor and Reviewers' comments. This work was supported by the China Scholarship Council, CONACyT(Mexico) and national "Robotex" project.

${ }^{1}$ Qiuyue Luo, Victor De-León-Gómez, Anne Kalouguine, Christine Chevallereau and Yannick Aoustin are with Laboratoire des Sciences du Numerique de Nantes (LS2N), CNRS, Centrale Nantes, Universite de Nantes, Nantes, France, qiuyue. luo@ls2n.fr

${ }^{2}$ Anne Kalouguine is with Softbank Robotics

Digital Object Identifier (DOI): see top of this page. objective is to produce a control strategy that does not require online foot placement calculation based on prediction and/or optimization since we assume that humans' walking on even grounds does not require attention. Different from [8], the step timing applied in this paper is not explicitly but implicitly adapted by the transition between steps based on the CoM position.

We call the control approaches where several online adjustments for future foot locations must be done for each step high-level control. This includes methods based on prediction (or preview control), online optimization, and event-based control [9] that updates the parameters of the controller to stabilize the walking. The notions self-synchronization and selfstabilization are used when synchronization or stabilization of walking gaits can be achieved without high-level control. Hamed Razavi et al. first introduced the notion of synchronization for the 3D LIP model in 2015 [10], which refers to the periods of the pendular motions in the sagittal and frontal planes tending to a common period. An appropriate definition of the support change on the 3D LIP model would induce selfsynchronization of the walking. In [11], H. Razavi et al. introduced an ellipse-shaped switching manifold to achieve selfsynchronization. Inspired by this work, this paper proposed a new switching manifold that works for a more general choice of swing foot locations, and introduces a phasing variable to coordinate all the joint motions. Besides synchronization, walking stability of robots is another dominant feature to be analyzed. In this paper, a slight modification of the switching manifold is proposed to achieve self-stabilization.

The objective of this paper is to find some physical conditions that lead to self-synchronization or self-stabilization based on a simplified model. For this we will study different conditions to switch the stance leg, based on time or on the configuration of the robot. At the same time, different landing positions of the swing foot are also studied. This paper proves that self-synchronization is not observed when the switching condition is based on time or when both the step length and width are fixed, i.e. independent from the CoM position. On the other hand, self-synchronization can be obtained when the switching condition is based on a linear combination of the CoM positions along the sagittal and frontal axes. Moreover, it has been proved that self-stabilization can be obtained when the CoM velocity feedback in the sagittal plane is taken into account.

The paper is structured as follows. In Section II, the hybrid model of the LIP is detailed. In Section III, the reference trajectory of the swing foot is introduced. In Section IV, the instability of the periodic motion in the case when the transition 


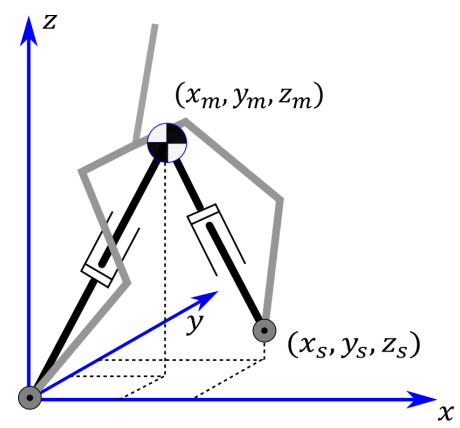

Fig. 1: A simplified model of a 3D biped robot.

is based on time is presented. In Section V and Section VI, switching conditions that lead to self-synchronization and selfstabilization of the walking gait are proposed . Finally, several concluding remarks are discussed in Section VII.

\section{Modeling OF THE WALKING GAIT VIA LIP MODEL}

\section{A. General description.}

In 3D LIP model, the robot is approximated as a point mass with point feet and the CoM trajectory is constrained to a plane for $3 \mathrm{D}$ walking. In this paper, the CoM trajectory is constrained to a horizontal plane. The gait is composed of two phases: single support phase and double support phase. During the double support phase, the following hypotheses are imposed: 1) The double support phase is instantaneous; 2) The contact between the swing leg and the ground does not modify the velocity of the CoM [12].

\section{B. Model in single support phase.}

In Fig. 1, a simplified model of a 3D biped robot is illustrated. Each leg is massless and has variable length. At the contact point, the stance leg rotates passively around $x$ and $y$ axes, the rotation around $z$ axis is not considered since this rotation is usually inhibited by friction in normal biped locomotion. There are two degrees of freedom at the hip, allowing to control the positions of the swing foot along $x$ and $y$ axes. The rotation along the leg axis is not considered since it does not modify the positions of the swing foot.

The configuration of the robot is defined via the CoM positions $\left(x_{m}, y_{m}, z_{m}\right)$ and the swing foot positions $\left(x_{s}, y_{s}, z_{s}\right)$ with respect to the reference frame attached to the stance foot. In order to explore simultaneously the self-synchronization and self-stabilization of periodic orbits for many step length and width, a dimensionless dynamic model of the pendulum will be used. The normalized scaling factors applied along $x$ and $y$ axes depend on the desired step length $S$ and desired step width $D$. Thus, a new set of variables is defined as: $\left(X, Y, z_{m}, X_{s}, Y_{s}, z_{s}\right)=\left(\frac{x_{m}}{S}, \frac{y_{m}}{D}, z_{m}, \frac{x_{s}}{S}, \frac{y_{s}}{D}, z_{s}\right)$.

For a pendulum with a constant height of CoM, the motions in the sagittal and frontal planes are decoupled. Thus, the equations of motion of the 3D LIP with respect to the reference frame attached to the stance foot are [1]:

$$
\begin{aligned}
& \ddot{X}=\omega^{2} X \\
& \ddot{Y}=\omega^{2} Y
\end{aligned}
$$

where $\omega=\sqrt{g / z_{m}}$ characterizes the LIP. As the legs of the robot are assumed to be massless, the swing leg motions $X_{s}, Y_{s}, z_{s}$ do not affect the dynamic equations of the 3D LIP.

The synchronization measure

$$
L=\dot{X} \dot{Y}-\omega^{2} X Y
$$

is conserved during a single support phase [10]. The walking gait is self-synchronized if and only if the synchronization measure is zero. In fact, this condition $L(X, Y, \dot{X}, \dot{Y})=0$ defines a sub-manifold. Any solution starting from this submanifold is self-synchronized and leads to periodic motion. It should be noted that the robot velocity is not fixed with the notion of self-synchronization.

\section{Transition between steps.}

Due to the hypothesis that the contact between the swing foot and the ground does not affect the velocity of the CoM, the velocity of CoM will be conserved at each transition of stance leg. Since the reference frame is always attached to the stance foot and the $y$ axis is directed toward the CoM, the sign of the velocity along $y$ axis will be changed from positive to negative [11], i.e.

$$
\begin{aligned}
& \dot{X}_{k+1}^{+}=\dot{X}_{k}^{-}, \\
& \dot{Y}_{k+1}^{+}=-\dot{Y}_{k}^{-} .
\end{aligned}
$$

The state before the transition, i.e. at the end of a step, is expressed by superscript ${ }^{-}$and that after the transition, i.e. at the beginning of a step, is expressed by ${ }^{+}$. The variables corresponding to the step $k$, are denoted with index $k$, while those of the next step are denoted with $k+1$.

After transition, the swing foot placement becomes the new stance foot placement. Thus the CoM position after transition along $x$ axis equals to the CoM position before transition minus the swing foot position. Similar result can be obtained for the CoM position along $y$ axis:

$$
\begin{aligned}
& X_{k+1}^{+}=X_{k}^{-}-X_{s, k}^{-} \\
& Y_{k+1}^{+}=-Y_{k}^{-}+Y_{s, k}^{-}
\end{aligned}
$$

Knowing the final state of the single support phase, the transition model (3) and (4) determines the initial state of the ensuing single support phase.

\section{Hybrid model.}

The transition is assumed to occur when the height of the swing foot $z_{s}$ is zero. A switching manifold is defined below:

$$
\mathscr{S}:=\left\{\mathbf{x} \mid z_{s}=0, \dot{z}_{s}<0\right\}
$$

where $\mathbf{x}:=\left[X, Y, z_{m}, X_{s}, Y_{s}, z_{s}, \dot{X}, \dot{Y}, \dot{z}_{m}, \dot{X}_{s}, \dot{Y}_{s}, \dot{z}_{s}\right]^{\top}$ is the state of the robot. The transition model (3) and (4) can be rewritten as:

$$
\mathbf{x}^{+}=\Delta\left(\mathbf{x}^{-}\right)
$$

where $\Delta$ indicates the transition map. Thus the combination of the dynamic equations and the transition model (6) forms the single-phase hybrid dynamical system:

$$
\Sigma: \begin{cases}\dot{\mathbf{x}}=f(\mathbf{x})+g(\mathbf{x}) u, & \mathbf{x}^{-} \notin \mathscr{S} \\ \mathbf{x}^{+}=\Delta\left(\mathbf{x}^{-}\right), & \mathbf{x}^{-} \in \mathscr{S}\end{cases}
$$


where $u$ is the control law that allows us to track the reference trajectory of the swing foot and keep the height of the CoM to be constant.

\section{E. Periodic motion.}

For a normalized system, periodic symmetric motion varies from $\left(X^{*+}, Y^{*+}\right)=\left(-\frac{1}{2}, \frac{1}{2}\right)$ to $\left(X^{*-}, Y^{*-}\right)=\left(\frac{1}{2}, \frac{1}{2}\right)$, where the superscript $*$ denotes the periodic motion. Since the orbital energy [13] is conserved during one step, the norm of the velocity at the beginning and end of a step is conserved, while the sign of it along $y$ axis is changed due to the change of reference frame:

$$
\begin{array}{lll}
\dot{X}^{*-} & =\dot{X}^{*+}, \\
\dot{Y}^{*-} & = & -\dot{Y}^{*+}
\end{array}
$$

Thus, the initial velocity of the CoM for a periodic motion with duration $T$ can be pointed out by solving equation (1):

$$
\begin{aligned}
& \dot{X}^{*+}=\omega \frac{1+\cosh (\omega T)}{2 \sinh (\omega T)}, \\
& \dot{Y}^{*+}=\omega \frac{1-\cosh (\omega T)}{2 \sinh (\omega T)} .
\end{aligned}
$$

We characterize the orientation of the velocity at the end of the single support phase by $\alpha=\frac{\dot{Y}^{*-}}{\dot{X}^{*-}}=-\frac{\dot{Y}^{*+}}{\dot{X}^{*+}}$. For a periodic motion in normalized coordinates $0<\alpha<1$.

\section{THE SWING FOOT MOTION}

\section{A. The phasing variable}

A normalized variable $\Phi$ monotonically increasing from 0 to 1 during one step, named phasing variable is defined to describe the desired trajectory of the controlled variables. For the case when transition is based on time, $\Phi$ is the time normalized with respect to the desired step duration. And for the case when transition is based on the CoM position, $\Phi$ is a function of $X$ and $Y$. The trajectories of the swing foot are defined as a function of $\Phi: X_{s}=X_{s}(\Phi), Y_{s}=Y_{s}(\Phi)$, $z_{s}=z_{s}(\Phi)$. The vertical evolution of swing foot determines the step timing while the horizontal evolution determines foot locations. The advantage of introducing virtual constraints based on a phasing variable is that this method can be extended to a complete model of robots by using the phasing variable to coordinate all the joint motions of the robot.

\section{B. The vertical swing foot motion.}

An intermediate value of the phasing variable $0<\Phi_{m}<1$ is defined, so that when $\Phi=\Phi_{m}, h_{z}=\max _{0<\Phi<1}\left\{z_{s}\right\}$. For the vertical motion of swing foot, the boundary conditions are:

$$
\begin{aligned}
& z_{s}(0)=0, \quad z_{s}\left(\Phi_{m}\right)=h_{z}, \\
& \dot{z}_{s}(0)=0, \quad \dot{z}_{s}(1)=0, \\
& \left.\Phi_{m}\right)=0, \quad \dot{z}_{s}(1)=v_{z},
\end{aligned}
$$

where $h_{z}$ is the desired height of the swing foot when $\Phi=$ $\Phi_{m}$, and $v_{z}<0$ denotes the desired downward velocity of the swing foot at the end of a step. In this paper, $z_{s}(\Phi)$ is defined as a cubic spline.

\section{The horizontal swing foot motion.}

The position where to land the swing foot must be chosen cautiously. In order to analyze several cases, the swing foot positions at the end of step $k$ is expressed in a generalized form:

$$
\begin{aligned}
& X_{s, k}^{-}=\left(1-k_{S}\right)\left(X_{k}^{-}-X^{*-}\right)+1 \\
& Y_{s, k}^{-}=\left(1-k_{D}\right)\left(Y_{k}^{-}-Y^{*-}\right)+1
\end{aligned}
$$

where $0 \leq k_{S} \leq 1$ and $0 \leq k_{D} \leq 1$. How the parameters $k_{S}$ and $k_{D}$ affect the foot locations is illustrated in Fig. 2. The case $k_{S}=k_{D}=0$ allows to nullify the CoM position error at the beginning of the next step, i.e. $\delta X_{k+1}^{+}=X_{k+1}^{+}-$ $X^{*+}=0, \delta Y_{k+1}^{+}=Y_{k+1}^{+}-Y^{*+}=0$. The case $k_{S}=k_{D}=1$ corresponds to fixed step length and width.

The boundary conditions for the motion of the swing foot along $x$ direction $X_{s}(\Phi)$ are:

$$
\begin{array}{ll}
X_{s}(0)=X_{s, k}^{+}, & \dot{X}_{s}(0)=0, \\
X_{s}(1)=X_{s, k}^{-}, & \dot{X}_{s}(1)=0,
\end{array}
$$

where $X_{s, k}^{+}$is the swing foot position at the beginning of step $k$, and it can be known according to the information of the previous step.

The same constraints are imposed on the motion along $y$ direction $Y_{s}(\Phi)$. With these boundary conditions, the trajectory along $x$ and $y$ directions can be defined as a third order polynomial functions of $\Phi$.

\section{TRANSITION BASED ON TIME}

In this section, the phasing variable is defined as $\Phi=\frac{t}{T^{*}}$, where $T^{*}$ is the desired step duration.

\section{A. Stability study.}

The classical technique for determining the existence and local stability properties of periodic orbits in nonlinear systems involves Poincaré return maps [14], [15]. The Jacobian matrix of the Poincaré return map at the fixed point is calculated numerically in the coordinate system $\left(X_{k}^{-}, Y_{k}^{-}, L_{k}^{-}, K_{k}^{-}\right)$, where $L_{k}^{-}$is the synchronization measure and $K_{k}^{-}$is the kinetic energy at the end of step $k$. Synchronization is characterized by all the eigenvalues associated to $X_{k}^{-}, Y_{k}^{-}, L_{k}^{-}$less than 1 and the eigenvalue associated to $K_{k}^{-}=1$ [11].

The eigenvalues of several cases for different values of $k_{D}$ and $k_{S}$ have been studied, and two extreme cases $k_{S}=k_{D}=$ 0 and $k_{S}=k_{D}=1$ among them are illustrated in Fig. 3. It can be seen clearly that for both cases, there are always more than one eigenvalue larger than or equals to one, which means that for the transition based on time, self-synchronization and thus self-stabilization, are not achieved.

\section{B. Simulation}

One example of simulation for $k_{S}=k_{D}=0$ starting slightly out of the periodic motion is analyzed here. The step length $S$ and step width $D$ are $0.4 \mathrm{~m}$ and $0.2 \mathrm{~m}$ respectively, and the height of the $\operatorname{CoM} z_{m}$ is $1 \mathrm{~m}$. The desired step duration 

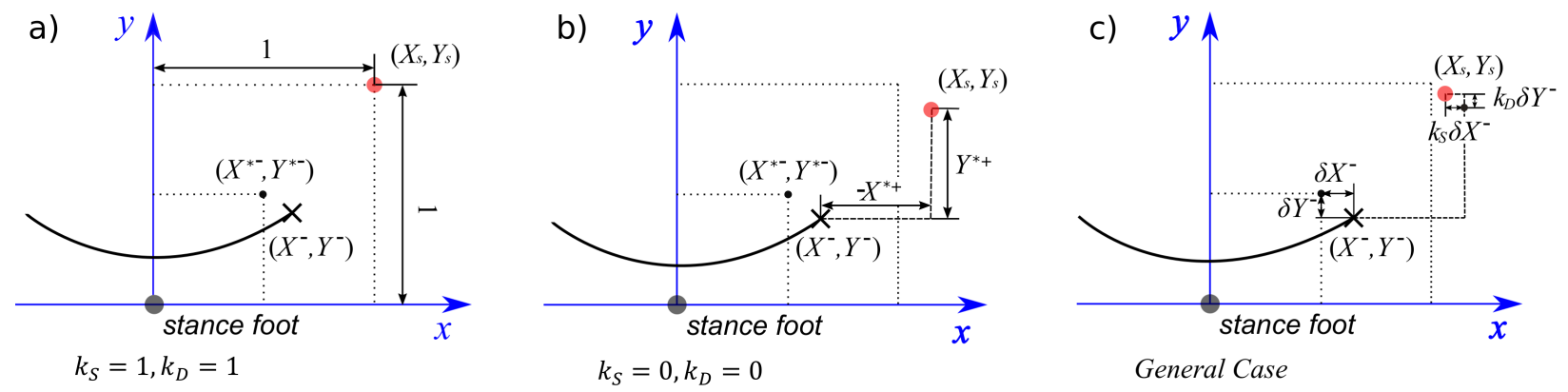

Fig. 2: Influence of $k_{S}$ and $k_{D}$ on the foot locations. a) Step length and width are fixed; b) The initial CoM position error is nullified; c) The general case. The black and the red dots represent respectively the stance feet during the current and the next steps. The curved line represents the CoM trajectory, and the cross the CoM position at the end of the current step.
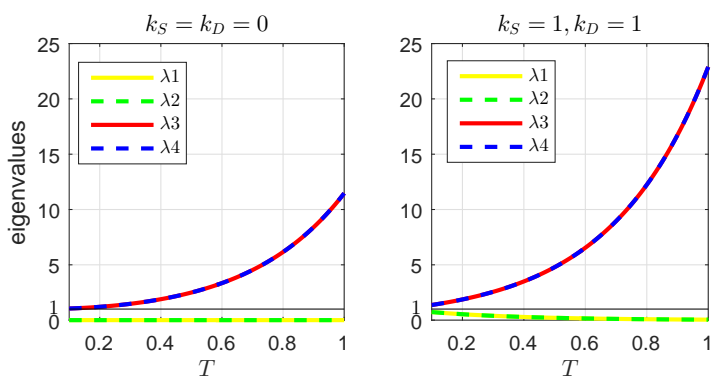

Fig. 3: Values of the four eigenvalues for different step duration $T$ when transition is based on time.

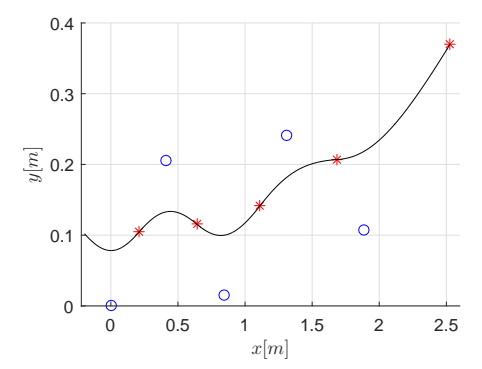

Fig. 4: Evolution of CoM of the LIP model when transition is based on time for $k_{S}=k_{D}=0$. The blue dots represent the stance feet and the red asterisks the CoM positions at the end of each step.

is set to be $0.6 \mathrm{~s}$. The eigenvalues calculated for the linearized restricted Poincaré map are:

$$
\begin{aligned}
& \left|\lambda_{1}\right|=\left|\lambda_{2}\right|=0, \\
& \left|\lambda_{3}\right|=\left|\lambda_{4}\right|=2.497 .
\end{aligned}
$$

The stance foot position and the CoM evolution are illustrated in Fig. 4. In this case, it can be seen clearly that the CoM position diverges quickly.

In consequence, this kind of control is not naturally synchronized and a high-level control must be added to produce a synchronized or stable walking gait. In the following sections, we will prove that by defining proper virtual constraints and a phasing variable depending on the state of the robot, selfsynchronization and self-stabilization of the walking gait can

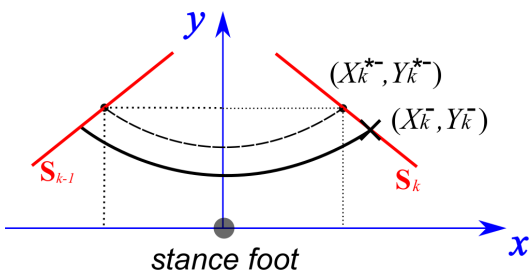

Fig. 5: The step finishes when the CoM crosses the switching manifold. The dashed line is the periodic motion, and the solid line the CoM motion under an initial position perturbation.

be obtained.

\section{TRANSITION BASED ON THE COM POSITION}

\section{A. The virtual constraints}

The vertical trajectory of the swing foot is defined as a function of $\Phi(X, Y)$. The transition happens when the swing foot touches the ground, which defines a relationship between the two variables $X$ and $Y$. An infinite number of CoM positions satisfy it. This set of positions are grouped in the switching configuration manifold defined by:

$$
\mathbb{S}=\left\{(X, Y) \mid z_{s}(\Phi)=0\right\} .
$$

In this paper, we choose a phasing variable such that the robot switches its stance leg when the CoM crosses the switching manifold:

$$
\mathbf{S}=\left\{(X, Y) \mid\left(X-X^{*-}\right)+C\left(Y-Y^{*-}\right)=0\right\} .
$$

The switching manifold $\mathbf{S}$ is defined as a line parameterized by $C$, represented by the red line in Fig. 5. Many other sets of positions can be considered but since stability studied here is a local property, a straight line is a convenient choice. The choice of $\mathbf{S}$ directly affects the final CoM position for step $k$.

\section{B. The phasing variable}

If $\Phi$ is expressed as a linear expression of $X$ and $Y$, no monotonic evolution of $\Phi$ has been found numerically for a periodic gait of the 3D LIP. Therefore, a quadratic expression of $X$ and $Y$ is proposed, i.e. 


$$
\Phi=a X+b Y+c X Y+d X^{2}+e Y^{2}+f .
$$

Since the phasing variable must be zero at the beginning of a step, $\Phi\left(X^{+}, Y^{+}\right)=0$, where $X^{+}$and $Y^{+}$are known from equation (4). Besides, in order to make the switching manifold (14) and (15) equivalent, the phasing variable at the end of a step $\Phi\left(X^{-}, Y^{-}\right)=1, X^{-}=X^{*-}-C \delta, Y^{-}=Y^{*-}+\delta$, where $\delta$ is the CoM position error along $y$ axis at the end of a step. Since all the above constraints must be satisfied for an arbitrary value of $\delta$, three more equations can be obtained and we have:

$$
\left\{\begin{aligned}
a X^{+}+b Y^{+}+c X^{+} Y^{+}+d\left(X^{+}\right)^{2}+e\left(Y^{+}\right)^{2}+f & =0 \\
a X^{*-}+b Y^{*-}+c X^{*-} Y^{*-}+d\left(X^{*-}\right)^{2}+e\left(Y^{*-}\right)^{2}+f & =1 \\
-c C+d C^{2}+e & =0 \\
-a C+b-c C Y^{*-}+c X^{*-}-2 d C X^{*-}+2 e Y^{*-} & =0 .
\end{aligned}\right.
$$

There are six variables $\{a, b, c, d, e, f\}$ while the number of equations is only four. For simplification, the value of $c$ is set to be 0 , thus $\Phi$ is expressed as a function of $d$. After calculation, the phasing variable can be rearranged as:

$$
\begin{gathered}
\Phi=\frac{M_{1}}{M_{2}}+d M_{3} M_{4} \\
M_{1}=X-X^{+}+C Y-C Y^{+} \\
M_{2}=X^{*-}-X^{+}+C Y^{*-}-C Y^{+} \\
M_{3}=X-X^{*-}+C Y-C Y^{*-} \\
M_{4}=X-X^{+}-C Y+C Y^{+} .
\end{gathered}
$$

During a step, the phasing variable must be monotonic, thus the derivative of $\Phi$ with respect to time should always be positive. Monotonicity of $\Phi$ for the periodic motion, i.e. when $X^{+}=X^{*+}, Y^{+}=Y^{*+}$, is analyzed. A set of $d$ varying from -0.4 to -0.7 is chosen to satisfy the condition of monotonicity. The minimum values of $\dot{\Phi}$ for different values of $C, T$ and $d$ are shown in Fig. 6. Obviously, when the minimum value of $\dot{\Phi}$ is larger than zero, $\Phi$ is monotonically increasing. From the result shown in Fig. 6, $d=-0.4$ is taken for the following simulation because it allows a larger range of acceptable values of $C$ with respect to different values of $T$.

\section{Stability study.}

To study the stability of the walking gait, the Poincare return map is used. Since $X_{k}^{-}$and $Y_{k}^{-}$are coupled via the switching manifold (15), and the dynamic is autonomous (the time does not appear), the chosen independent state variables are $\left(X_{k}^{-}, L_{k}^{-}, K_{k}^{-}\right)$. The Jacobian is defined for a given periodic motion, and is characterized by the direction of the velocity of the CoM $\alpha$.

1) General case: The expression of the Jacobian matrix of Poincaré return map in an analytical form can be deduced as follows:

$$
J=\left[\begin{array}{ccc}
-\frac{k_{D}+\alpha C k_{S}}{\left(k_{S}+\alpha C\right)} & \frac{4 \alpha C k_{S}}{\omega^{2}(1+\alpha C)(\alpha-1)} & 0 \\
J_{21} & \frac{2 \alpha\left(k_{D}-C k_{S}\right)+(1-\alpha C)(\alpha+1)}{(1+\alpha C)(\alpha-1)} & 0 \\
* & * & 1
\end{array}\right],
$$
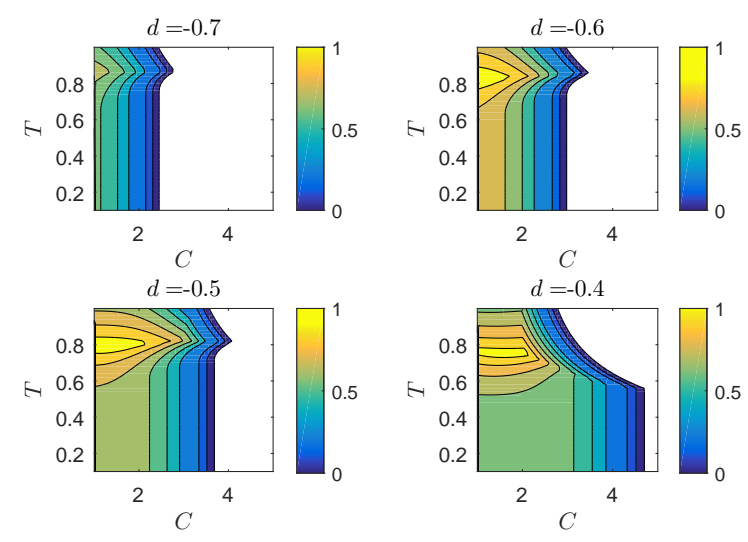

Fig. 6: The minimum value of $\dot{\Phi}$ as a function of $C$ and $T$ for different $d$. The colored areas, with respect to the white areas, indicate that the phasing variable is monotonic.

with $J_{21}=\frac{\left(k_{D}+\alpha C k_{S}\right)\left(C-1+C k_{S}-k_{D}\right) \omega^{2}}{2(1+\alpha C) C k_{S}}$. The two elements denoted by $*$ do not affect the eigenvalues. The eigenvalue associated with $K_{k}^{-}$is always 1 , and this means that the walking velocity cannot be controlled. It is possible to find a proper set of parameters $C, k_{S}$ and $k_{D}$ to achieve selfsynchronization. A numerical calculation of the largest norm of the eigenvalues associated to the $2 \times 2$ left-upper part of (20), for different values of $k_{D}$ and $k_{S}$ is shown in Fig. 7. The main conclusion is that when $k_{D}$ and $k_{S}$ decrease, the norm of the eigenvalues is smaller for an appropriate choice of $C$, for any $T \in(0,1] \mathrm{s}$. One particular set of $k_{D}, k_{S}$ that will not lead to self-synchronization is considered below.

2) Particular case $k_{D}=k_{S}=1$ : In this case, the Jacobian matrix (20) becomes

$$
J=\left[\begin{array}{ccc}
-1 & \frac{4 \alpha C}{\omega^{2}(1+\alpha C)(\alpha-1)} & 0 \\
\frac{(C-1) \omega^{2}}{C} & \frac{2 \alpha(1-C)+(1-\alpha C)(\alpha+1)}{(1+\alpha C)(\alpha-1)} & 0 \\
* & * & 1
\end{array}\right]
$$

The eigenvalues of the Jacobian matrix are $\lambda_{1}, \lambda_{2}=$ $\frac{(1+\alpha)(1-\alpha C) \pm 2 \sqrt{\alpha(C-1)\left(\alpha^{2} C-1\right)}}{(1+\alpha C)(\alpha-1)}, \lambda_{3}=1$. When $\alpha(C-$ 1) $\left(\alpha^{2} C-1\right)<0$, it can be proven that all the norms of the eigenvalues are strictly equal to 1 . In the other case at least one eigenvalue has a norm greater than 1. In both cases, no self-synchronization can be achieved.

The case $k_{S}=k_{D}=0$ is especially interesting since the eigenvalues of the Jacobian matrix can be very small for an appropriate choice of $C$. This case has been explored in [16]. One drawback of this choice is the lateral error that can appear on the walking path due to the variation of the step width to nullify the error along $y$ direction. In the following part of the paper, $k_{S}=0, k_{D}=1$ will be chosen, because $k_{S}=0$ can contribute to achieve self-synchronization while $k_{D}=1$ can prevent the robot from deviating from its original direction. 

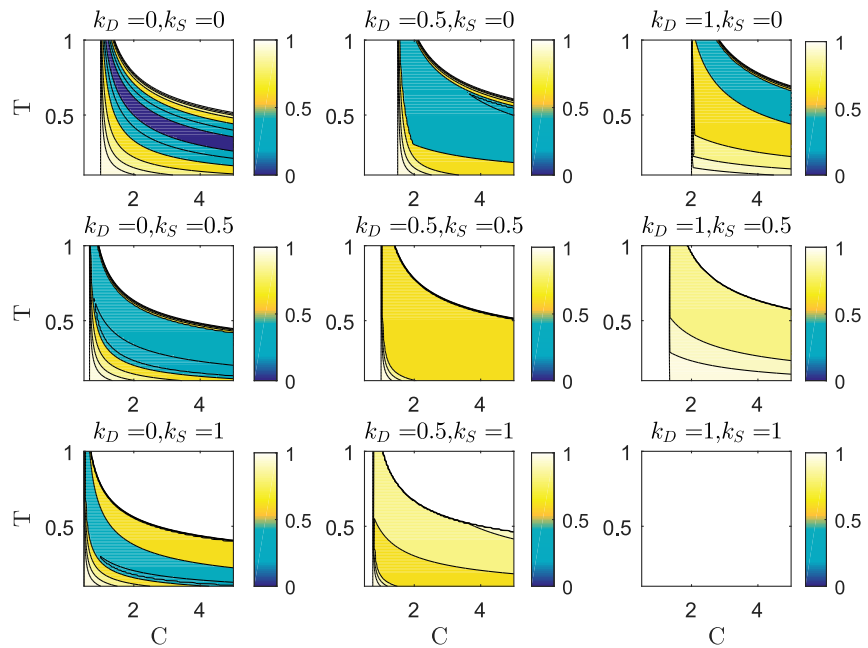

Fig. 7: Numerical calculation of the largest norm of the eigenvalues associated to the $2 \times 2$ left-upper part of (20), for different values of $k_{D}$ and $k_{S}$

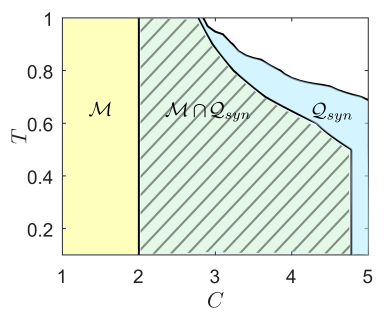

Fig. 8: Intersection of $\mathcal{Q}_{\text {syn }}$ and $\mathcal{M}$ for $k_{S}=0, k_{D}=1$

\section{Choice of $C$.}

The manifold of $C$ and $T$ that makes the walking gait selfsynchronized is defined as

$$
\mathcal{Q}_{\text {syn }}:=\left\{(C, T) \mid \lambda_{1,2}(C, T)<1, \lambda_{3}(C, T)=1\right\} .
$$

Meanwhile, the manifold of $C$ and $T$ that makes the phasing variable $\Phi$ monotonic for the periodic gait is defined as

$$
\mathcal{M}:=\left\{(C, T) \mid \min _{0 \leq t \leq T}\{\dot{\Phi}(C, T)\}>0\right\} .
$$

Thus, in order to accomplish a step, the values of $C$ and $T$ must be located inside of the intersection of $\mathcal{Q}_{\text {syn }}$ and $\mathcal{M}$, i.e. $(C, T) \in \mathcal{Q}_{\text {syn }} \cap \mathcal{M}$. Fig. 8 shows the acceptable values of $C$ and $T$ for $k_{S}=0, k_{D}=1$.

\section{E. Simulation}

The step length $S$ and step width $D$ are $0.4 \mathrm{~m}$ and $0.2 \mathrm{~m}$ respectively, and the height of the $\mathrm{CoM} z_{m}$ is $1 \mathrm{~m}$. The desired step duration is 0.6s. According to Fig. 8, the chosen value of $C$ is 3. The walking algorithm proposed by S. Kajita in [1] is integrated in the simulation for the first and the last steps and is not explained in detail in this paper, because it does not affect the self-synchronization or self-stabilization of the periodic motion.

Fig. 9a shows the evolution of the CoM and the footprints for 15 steps, and the convergence to a periodic gait motion

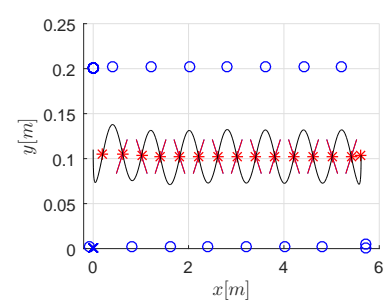

(a) Evolution of the CoM

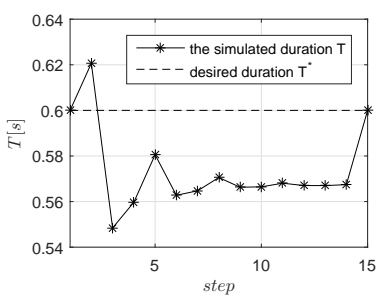

(b) Evolution of $T$
Fig. 9: 15 steps for the LIP model when transition is based on the CoM position. The red lines in (a) represent the switching manifolds, the blue dots the stance feet, and the red asterisks the CoM positions at the end of each step.
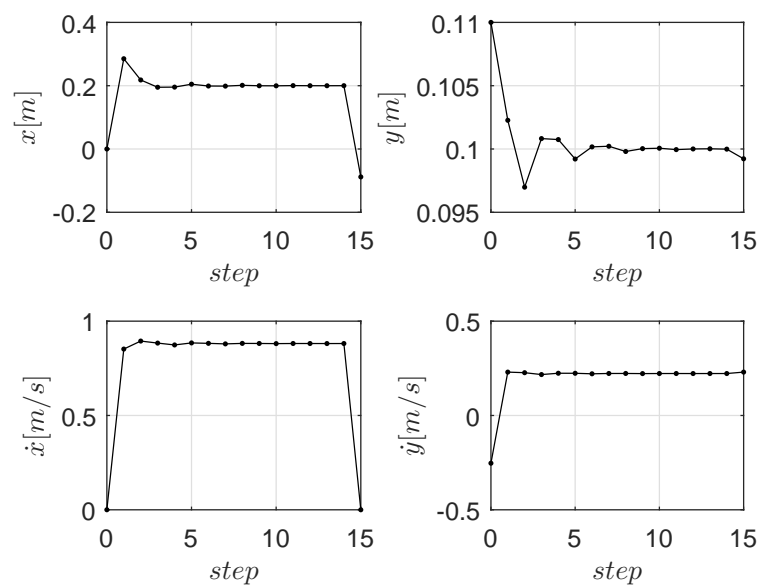

Fig. 10: Evolution of the states of the CoM when transition is based on the CoM position

is observed. However, the actual step duration $T$ does not converge to the desired one $T^{*}$, as shown in Fig. 9b. It means that the walking velocity of the gait is not controlled in the sense that a perturbation of such a gait will result in the convergence of the gait to another periodic motion with a different gait velocity. At the first and the last steps, the step duration is $0.6 \mathrm{~s}$ due to the application of the walking algorithm proposed by Kajita. From Fig. 10, the states of the CoM in the poincaré section converge to constant values, which means that the walking gait is self-synchronized but not self-stabilized.

\section{TRANSITION BASED ON THE COM POSITION AND VELOCITY FEEDBACK}

In a previous work [17], vertical oscillation of the CoM, with a downward velocity at the step transition, was introduced to transform a self-synchronized walking gait into a selfstabilized one. By using that method, the motion of the robot is more human-like but the LIP model is no longer valid. This brings difficulties such as the finding of the periodic motion and the integration of starting/stopping phases. Thus, a new method is introduced so that the LIP model will still be valid. 


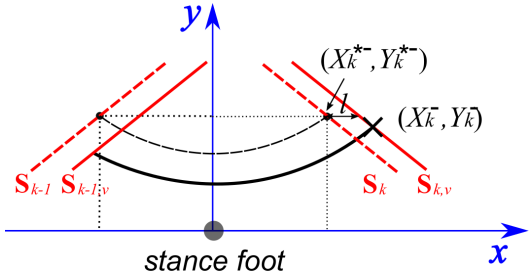

Fig. 11: The new switching condition is an offset from the one proposed in last section. The dashed line is the periodic motion, while the solid line is the CoM motion under an initial position perturbation.

\section{A. The virtual constraints}

The feedback of the velocity of CoM is introduced into the switching condition of the stance leg, and a new switching manifold is proposed:

$$
\mathbf{S}_{\mathbf{v}}=\left\{(X, Y) \mid\left(X-X^{*-}-l\right)+C\left(Y-Y^{*-}\right)=0\right\}
$$

As shown in Fig. 11, the new switching manifold is a line with an offset $l$ from the switching condition proposed in last section. The offset is defined as $l=k_{v}\left(\dot{X}^{*+}-\dot{X}_{k}^{+}\right)$, where $\dot{X}_{k}^{+}$is the velocity of the CoM along $x$ axis at the beginning of step $k$, which is updated at each step ${ }^{3}$, and $k_{v}$ is a parameter that must be chosen carefully to satisfy the stability condition.

\section{B. The phasing variable}

With the CoM velocity feedback along $x$ axis, the CoM position at the end of a step is modified, i.e. $X^{-}=X^{*-}-$ $C \delta+l, Y^{-}=Y^{*-}+\delta$. Thus the phasing variable $\Phi$ can be deduced by applying $X^{-}$and $Y^{-}$into $\Phi\left(X^{-}, Y^{-}\right)=1$ :

$$
\Phi=\frac{M_{1}+d M_{2} M_{3} M_{4}-d M_{4} l+d\left(M_{3}-M_{2}\right) M_{4} l^{2}}{M_{2}+l}
$$

By adjusting the position of the switching manifold, the difference of velocity between the current velocity and the desired velocity along $x$ direction can be controlled to obtain self-stabilization.

\section{Stability study}

With this new phasing variable, the stability of the gait is analyzed through the numerical calculation of the eigenvalues of the Jacobian matrix for different values of $C, k_{v}$ and $T$. The contour of the maximum eigenvalue is shown in Fig. 12. It can be seen that when both the values of $C$ and $k_{v}$ are located inside the colored area, all the eigenvalues will be smaller than one. Under this situation, the self-stabilization of the system can be obtained. Whereas in the white area at least one eigenvalue is larger than one.

\section{Choice of $C$.}

In order to make the walking gait stable, the manifold of $C$ and $T$ is defined as:

$$
\mathcal{Q}_{s t a b}:=\left\{(C, T) \mid \lambda_{i}<1(i=1, \cdots, n)\right\},
$$

\footnotetext{
${ }^{3}$ The correction can be based on the mean value of velocity during the previous step to increase the robustness to the measurement noise
}

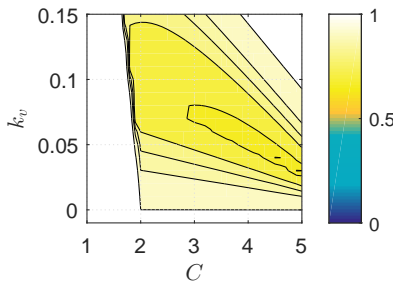

(a) $T=0.6 \mathrm{~s}$

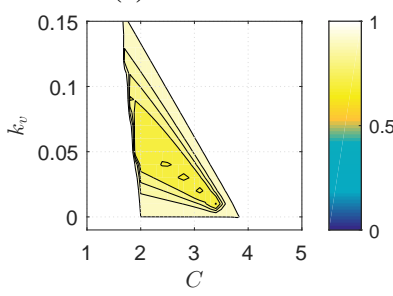

(c) $T=0.8 \mathrm{~s}$

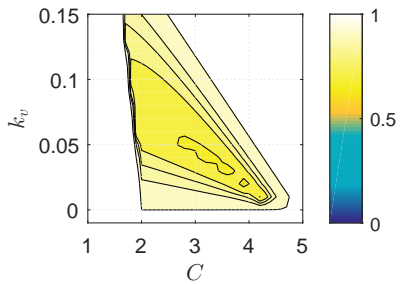

(b) $T=0.7 \mathrm{~s}$

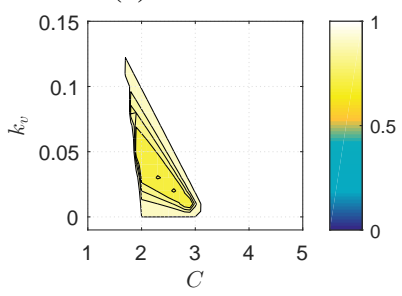

(d) $T=0.9 \mathrm{~s}$
Fig. 12: The maximum eigenvalue as a function of $C$ and $k_{v}$ for different values of $T$. The colored areas, with respect to the white areas, indicate the self-stabilization condition.

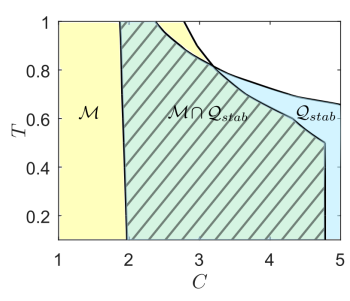

(a) $k_{v}=0.04$

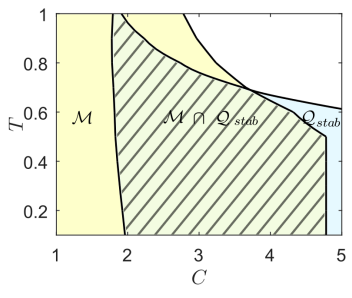

(b) $k_{v}=0.08$
Fig. 13: Intersection of $\mathcal{Q}_{s t a b}$ and $\mathcal{M}$ that keeps $\Phi$ monotonic and produces a stable walking gait for $k_{v}=0.04$ and $k_{v}=$ 0.08 respectively

where $n$ is the number of eigenvalues. Considering the fact that $C$ and $T$ must also satisfy the constraint that $\Phi$ is monotonic, the values of $C$ and $T$ must be located inside of the intersection of $\mathcal{Q}_{\text {stab }}$ and $\mathcal{M}$, i.e. $(C, T) \in \mathcal{Q}_{\text {stab }} \cap \mathcal{M}$. Fig. 13a and Fig. 13b show the acceptable values of $C$ and $T$ for $k_{S}=0, k_{D}=$ 1 when $k_{v}=0.04$ and $k_{v}=0.08$ respectively.

\section{E. Simulation}

The step length $S$ and step width $D$ are $0.4 \mathrm{~m}$ and $0.2 \mathrm{~m}$ respectively, and the height of the CoM $z_{m}$ is $1 \mathrm{~m}$. From the result shown in Fig. 13, $C=3$ is chosen. Based on the result shown in Fig. 12, $k_{v}=0.08$ when $T^{*}=0.6 \mathrm{~s}$, and $k_{v}=0.04$ when $T^{*}=0.8 \mathrm{~s}$ to ensure stability.

The same method proposed by S. Kajita as that in Section V-C is adopted for the first and last step. The desired step duration $T^{*}$ is increased from $0.6 \mathrm{~s}$ to $0.8 \mathrm{~s}$ at the $15^{\text {th }}$ step, in order to show the convergence of the walking gait in velocity.

Fig. 14a illustrates the evolution of the CoM and the placement of feet. Due to the increase of step duration, the amplitude of the motion of the CoM along $y$ direction increases obviously after 15 steps. And Fig. 14b indicates that the actual step duration $T$ starts from $0.6 \mathrm{~s}$, and increases 


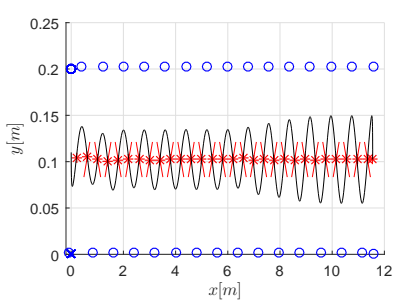

(a) Evolution of the CoM

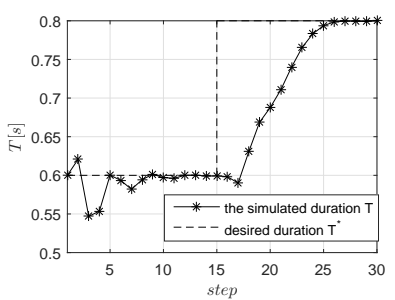

(b) Evolution of $T$
Fig. 14: Simulation of 30 steps for the LIP model when transition is based on the CoM position and velocity. The red lines in figure (a) represent the switching manifolds, the blue dots the stance feet, and the red asterisks the CoM positions at the end of each step.
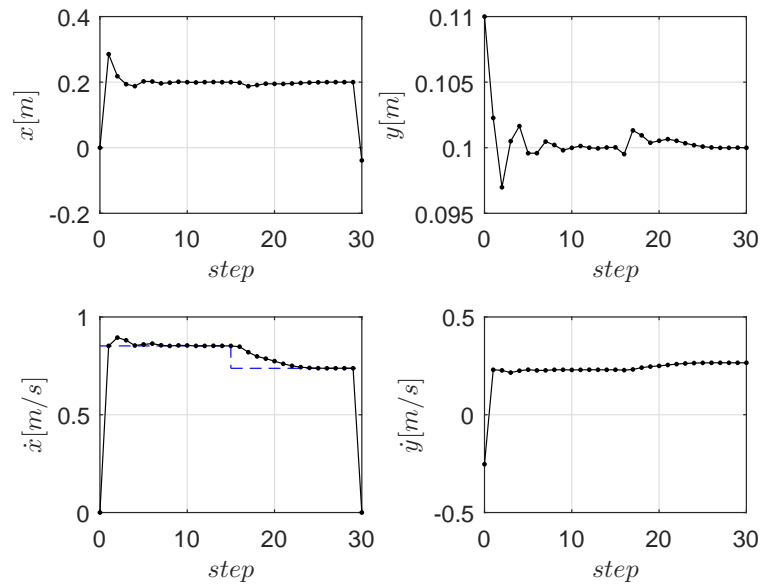

Fig. 15: Evolution of the states of the 3D LIP during 30 steps when transition is based on the CoM position and velocity. The desired CoM velocity at the end of a step along $x$ axis is shown with the dash line.

to $0.8 \mathrm{~s}$ after 15 steps. In Fig. 15 , it can be seen that the introduction of the displacement $l$ plays an important role during the motion. It permits that the velocity along $x$ axis converges to a desired value, i.e. self-stabilization is obtained.

\section{CONCLUSION}

In this paper, the switching conditions of selfsynchronization or self-stabilization of the periodic walking gait are discussed. It has been proved that self-synchronization cannot be achieved when the stance foot is switched as a function of time or when both the step length and width are fixed. In this case, synchronization and stabilization of walking gaits can be achieved only by using high-level control techniques. Thus this paper proposes to define the switching condition as a function of the CoM position to achieve self-synchronization. This condition is expressed as a linear function of the CoM position in a switching manifold parameterized by a parameter denoted by $C$. It is shown that for an appropriate value of $C$, if the swing foot placement is chosen such that the initial CoM position error is reduced step by step at least in one direction, synchronization of the walking can be naturally obtained. Furthermore, it has been proven that the introduction of the CoM velocity feedback into the switching condition permits to achieve self-stabilization of walking gaits.

\section{REFERENCES}

[1] S. Kajita, F. Kanehiro, K. Kaneko, K. Yokoi, and H. Hirukawa, "The 3d linear inverted pendulum mode: A simple modeling for a biped walking pattern generation," in Intelligent Robots and Systems, 2001. Proceedings. 2001 IEEE/RSJ International Conference on, vol. 1. IEEE, 2001, pp. 239-246.

[2] P.-B. Wieber, "Trajectory free linear model predictive control for stable walking in the presence of strong perturbations," in Humanoid Robots, 2006 6th IEEE-RAS International Conference on. IEEE, 2006, pp. 137-142.

[3] S. Kajita, F. Kanehiro, K. Kaneko, K. Fujiwara, K. Harada, K. Yokoi, and $\mathrm{H}$. Hirukawa, "Biped walking pattern generation by using preview control of zero-moment point," in 2003 IEEE International Conference on Robotics and Automation (Cat. No.03CH37422), vol. 2, Sept 2003. pp. 1620-1626 vol.2.

[4] J. Englsberger, C. Ott, M. A. Roa, A. Albu-Schäffer, and G. Hirzinger, "Bipedal walking control based on capture point dynamics," in Intelligent Robots and Systems (IROS), 2011 IEEE/RSJ International Conference on. IEEE, 2011, pp. 4420-4427.

[5] T. Koolen, T. De Boer, J. Rebula, A. Goswami, and J. Pratt, "Capturability-based analysis and control of legged locomotion, part 1: Theory and application to three simple gait models," The International Journal of Robotics Research, vol. 31, no. 9, pp. 1094-1113, 2012.

[6] T. Takenaka, T. Matsumoto, and T. Yoshiike, "Real time motion generation and control for biped robot-1 st report: Walking gait pattern generation," in Intelligent Robots and Systems, 2009. IROS 2009. IEEE/RSJ International Conference on. IEEE, 2009, pp. 1084-1091.

[7] J. Englsberger, C. Ott, and A. Albu-Schäffer, "Three-dimensional bipedal walking control based on divergent component of motion," IEEE Transactions on Robotics, vol. 31, no. 2, pp. 355-368, 2015.

[8] M. Khadiv, A. Herzog, S. A. A. Moosavian, and L. Righetti, "Step timing adjustment: A step toward generating robust gaits," in Humanoid Robots (Humanoids), 2016 IEEE-RAS 16th International Conference on. IEEE, 2016, pp. 35-42.

[9] J. Grizzle, "Remarks on event-based stabilization of periodic orbits in systems with impulse effects," in Second International Symposium on Communications, Control and Signal Processing, 2006.

[10] H. Razavi, A. M. Bloch, C. Chevallereau, and J. W. Grizzle, "Restricted discrete invariance and self-synchronization for stable walking of bipedal robots," in American Control Conference (ACC), 2015. IEEE, 2015, pp. $4818-4824$.

[11] — "Symmetry in legged locomotion: a new method for designing stable periodic gaits," Autonomous Robots, vol. 41, no. 5, pp. 11191142, 2017.

[12] S. Kajita, H. Hirukawa, K. Harada, and K. Yokoi, Introduction to humanoid robotics. Springer, 2014, vol. 101.

[13] S. Kajita, T. Yamaura, and A. Kobayashi, "Dynamic walking control of a biped robot along a potential energy conserving orbit," IEEE Trans. Robot. Autom., vol. 8, no. 4, pp. 431-438, 1992.

[14] A. Goswami, B. Espiau, and A. Keramane, "Limit cycles and their stability in a passive bipedal gait," in Robotics and Automation, 1996. Proceedings., 1996 IEEE International Conference on. IEEE, 1996, pp. 246-251.

[15] B. Morris and J. W. Grizzle, "A restricted poincaré map for determining exponentially stable periodic orbits in systems with impulse effects: Application to bipedal robots," in Decision and Control, 2005 and 2005 European Control Conference. CDC-ECC'05. 44th IEEE Conference on. IEEE, 2005, pp. 4199-4206.

[16] C. Chevallereau, H. Razavi, D. Six, Y. Aoustin, and J. Grizzle, "Selfsynchronization and self-stabilization of 3d bipedal walking gaits," Robotics and Autonomous Systems, vol. 100, pp. 43-60, 2018.

[17] C. Chevallereau and Y. Aoustin, "Self-stabilization of 3d walking via vertical oscillations of the hip," in Robotics and Automation (ICRA), 2015 IEEE International Conference on. IEEE, 2015, pp. 5088-5093. 